\title{
Further investigations of inhibitory mechanisms in attention
}

\author{
DOUGLAS G. LOWE \\ Trent University, Peterborough, Ontario, Canada
}

\begin{abstract}
Three experiments employed a discrete-trials version of the Stroop task to investigate further the claim that there are inhibitory mechanisms in selective attention (Neill, 1977). The results indicated that the evidence supporting the inhibitory theory (i.e., the diminished availability of distractor responses) cannot be explained by subjects' attempts to physically match successive stimuli (Lowe, 1979). In light of all the available evidence, it was suggested that the suppression effect does not index attentional inhibition, but may be attributed to a problem of code coordination (Keele \& Neill, 1978).
\end{abstract}

Deployment of selective attention permits an observer to respond optimally to chosen aspects of stimulation while excluding other, unwanted aspects. An important question concerns how this selective processing is achieved. Treisman (1964) hypothesized that selection of one message for analysis and response may sometimes involve the attenuation of others. According to this account, selection of some information may entail direct and specific inhibition of other information.

Evidence for the existence of some form of inhibition in the context of selective attention comes from recent experiments (Neill, 1977) employing the Stroop (1935) color-word task. The Stroop task requires subjects to name the ink color of a letter display while overcoming the tendency to read a different color name spelled by the letters. Employing a series of discrete trials, Neill (1977, Experiment 1) found that when two successive Stroop stimuli were related such that the color name of the second item (S2) matched the distracting word of the first (S1), observers took longer to respond than when the sequential inputs were unrelated (the suppression effect). Neill (1977) reasoned that when the color for S1 is named, the name of the conflicting color word is actively suppressed. Then, if that inhibited name is the correct response for $\mathbf{S 2}$, the inhibition slows responding, producing the suppression effect.

However, subsequent results did not support a simple inhibitory account. For example, Neill (1977, Experiment 2) found that $S 1$ distractor responses remained continuously available, and facilitated responses for $S 2$, when manual responding was substituted for vocal color naming. Lowe (1979, Experiment 3) also demonstrated a facilitatory effect during related trials when color patches

This research was supported by Grant A0284 from the National Sciences and Engineering Research Council of Canada. I am indebted to Sandra Thwaites and Bruce Tsuji for the aid in collecting and tabulating the present data. My mailing address is: Department of Psychology, Trent University, Peterborough, Ontario, Canada K9J 7B8. were substituted for color-word stimuli as S2. Lastly, results by Neill $(1977,1979)$ also indicated that inhibition varied with the relative demands for speed versus accuracy. More pronounced inhibitory effects were obtained when instructions emphasized accuracy of responding. It is evident that the suppression effect is extremely sensitive to a number of variations in experimental context. This phenomenon can be influenced by the nature of the response requirements as well as by other sequences and stimuli that appear in the task.

In light of these complications, Lowe (1979) suggested that subjects did not treat the successive stimuli as discrete, but employed preceding stimuli as predictors of subsequent items. Lowe argued that a number of transition effects, including the suppression effects, could be explained by a physical matching strategy in which, under certain circumstances, subjects compared the physical attributes of successive stimuli (Posner \& Mitchell, 1967). Lowe's matching argument was based on the premise that, in the Stroop task, subjects must use the available physical or visual information (i.e., ink-color and word-form) for each presented item in order to choose one color name over the other (Keele, 1973). Thus, following responses to S1, subjects' knowledge of the propriety of S1 ink-color and the impropriety of S1 word-form would enhance performance if both sources of information were directly compared with $\mathrm{S} 2$. The greater the physical similarity of the stimuli, the more performance would be improved.

For example, both Lowe (1979) and Neill (1978) observed that reaction times (RTs) were faster for conditions in which the colors of the stimuli were repeated than for trials in which the colors were changed (the repetition effect, Bertelson, 1963). In addition, the magnitude of the repetition effect was greater when both the colors and distractors were repeated than when only the colors were repeated, but the distractors were changed (the identity effect). To account for suppression effects, it was argued that subjects were slower to respond during related trials, not because the inappropriate response had been 
inhibited on S1, but rather because the S1 word was highly available and was included in the match. Thus, correct color names for S2, being concurrently associated with both a correct (color) and an incorrect (word) stimulus source, would be highly ambiguous until further, timeconsuming analysis had verified their propriety.

However, there are several difficulties in completely ascribing the pattern of results to physical matching. First, on the premise that subjects would be induced to compare the displays when the proportion of predictably valid S1 trials was high, Lowe (1979, Experiment 2) demonstrated that suppression effects were more likely to occur when color-repetition trials were more frequent than trials for which the colors were changed. However, Neill (1977, Experiment 1) obtained suppression effects when color-repetition trials were much less frequent than trials for which the colors were changed. If physical matching is the basis of the suppression effect, its occurrence should be consistently controlled by incentives to compare the displays. Second, in keeping with the matching hypothesis, Lowe (1979, Experiment 2) found that the magnitude of the repetition effect was greater when color-repetition trials were more frequent. However, the size of identity effects was insensitive to changes in the proportion of color-repetition trials. This finding suggests that identity effects are not governed by incentives to compare the displays. Finally, identity effects were also obtained by Neill (1977, Experiment 2, 1978, Experiment 1), but in those experiments, S1 distractor responses remained continuously available and facilitated responses for $\mathbf{S 2}$. This result suggests that identity and suppression effects are dissociable. Taken together, these findings provide difficulties for the notion that subjects' strategies to physically compare successive stimuli is the common source of repetition, identity, and suppression effects. Indeed, results reported below clearly reveal the independence of identity and suppression effects.

\section{EXPERIMENT 1}

In all previous experiments, the distractor words were always printed in uppercase letters. In light of Lowe's (1979) demonstration of the importance of a strategy of sequential dependencies, it is possible that subjects may have been encouraged to compare the sequential distractors. Thus, identity effects might have occurred independently of both suppression effects and incentives to match the colors of the displays.

In this experiment, identity and suppression effects were examined under conditions that were known to discourage physical comparison of sequential stimuli. The procedure followed that of Lowe (1979. Experiment 2), in which subjects were shown two successive color-word stimuli and were required to name the colors of both items. The main manipulation involved unpredictable alternations in the fonts of the letters in which the distractor words were printed. One-half of all trials consisted of sequences of similar-case distractor information (i.e.. uppercase/up- percase, lowercase/lowercase), whereas the other half consisted of different-case stimuli (i.e., uppercase/lowercase, lowercase/uppercase).

It has been shown that unpredictably mixing the fonts of sequentially presented alphabetic stimuli impairs physical matching strategies, as opposed to when the inputs are presented in the same font (pure lists) (Posner, Boies, Eichelman, \& Taylor, 1969). In the current paradigm, mixed lists may remove the incentive for subjects to compare successive distractors. Thus, identity effects may not be obtained or, perhaps, may be obtained only for same-case displays. ${ }^{1}$ Moreover, if, as suggested by Lowe's (1979) hypothesis, suppression effects can be attributed to comparison of successive word-forms, suppression effects may not be obtained in the absence of incentives to match distractor stimuli. Alternatively, if identity and suppression effects are independent, suppression effects may occur regardless of variations in the fonts of the inputs.

\section{Method}

Design and Materials. Twenty examples of 12 different Stroop stimuli were prepared for presentation in a tachistoscope by printing the words "red," "blue," "green," and "yellow" on white cards in 28-point Letraset Projectatype (PT 102). The four words were printed in each of the above-named colors, except for that color congruent to the named word. The visual angles subtended by the different stimuli varied from approximately $2.34^{\circ}$ to $5.22^{\circ}$. One-half of the examples were printed in uppercase letters. whereas the other half were printed in lowercase letters. These stimuli were assigned to one of five packs of 48 items each. For each pack, one-half of the items consisted of uppercase stimuli, whereas the remainder consisted of lowercase materials. Within each pack, the items were ordered such that when Packs 1-2, 2-3, 3-4, and 4-5 were paired. four different experimental conditions resulted. as defined by the following relationships between the items: ( 1 ) color and word repeated (CAWR) - both the colors and distractors of the stimuli were identical [e.g., the word "red" (painted) in blue ink followed by $(\rightarrow)$ "red" in blue ink]; (2) color only repeated (COR)-only the colors of the stimuli were identical, the distractor words were changed (e.g., "green" in blue ink - "red" in blue ink): (3) control (CONT)-the names of the colors and distractors of the pair were completely unrelated (e.g. " "yellow" in green ink - "red" in blue ink); (4) color matches preceding word (CMPW) - the distractor for S1 and the color for S2 had identical names, and the names of the color for $S 1$ and the distractor for $S 2$ were unrelated (e.g., "blue" in green ink - "red" in blue ink).

Since Lowe (1979, Experiment 2) had established that, in this paradigm. suppression effects are more likely to occur when subjects are induced to compare the colors of the inputs, the items were ordered within the packs so that $75 \%$ of all trials consisted of CAWR and COR sequences $(37.5 \%$ each). Thus, for both similar- and dissimilar-case displays, CAWR and COR trials each constituted $18.75 \%$ of all trials; the other trials were made up of CONT and CMPW stimuli (6.25\% each). Stimuli for the odd-numbered packs appeared in the upper portion of the visual field, and those items for the even-numbered packs appeared in the lower portion. The separation between the near edges of the stimuli was approximately $1.44^{\circ}$ of visual angle when the displays were viewed simultaneously. Each subject received the four different pairings of the packs twice. and data from the first 96 trials were excluded from analysis.

Procedure. All stimulus materials were presented by means of a Gerbrand three-field tachistoscope. On each trial, the subjects first viewed a blank white preexposure field. They then initiated exposure of SI. Responses to SI concurrently (1) terminated SI presenta- 
tion, (2) initiated $S 2$ presentation, and (3) started a millisecond timer. Responses to $\mathbf{S 2}$ stopped the timer, and $\mathbf{S 2}$ remained in view for $1 \mathrm{sec}$ before being replaced by the blank white field. The subjects were instructed to name the colors of the successive items and to ignore the distracting words themselves.

Subjects. The eight subjects were male and female undergraduates at Trent University, they were paid $\$ 3.00$ each for their participation.

\section{Results and Discussion}

For each subject, median RTs were calculated for each condition for same- and different-case stimuli. Performance scores for those sequences that made up $6.25 \%$ and $18.75 \%$ of all trials were based on 18 and 54 , or fewer, correct trials, respectively. The RT scores were submitted to a $4 \times 2$ repeated-measures analysis of variance in order to evaluate conditions and case (same/different).

The analysis revealed that neither the effect of case $[\mathrm{F}(1,7)<1, \mathrm{MSe}=313]$ nor the interaction of case and condition $[\mathrm{F}(3,21)=1.47, \mathrm{MSe}=276]$ was statistically reliable. $^{2}$ There were large effects for RTs as a function of condition $[F(3,21)=92.6$, MSe $=3,226]$. For samecase displays, mean RTs for CAWR, COR, CONT, and CMPW sequences were 431, 439, 658, and $696 \mathrm{msec}$, respectively, whereas for different-case inputs, the respective values were $437,440,650$, and $693 \mathrm{msec}$. It is evident that average RTs for both CAWR and COR sequences were much faster than those for CONT stimuli, whereas RTs for CMPW trials were somewhat slower. Newman-Keuls comparisons of the means of the four conditions revealed the pattern: (CAWR, COR), (CONT), (CMPW), where only conditions within the same set of parentheses did not differ at the 5\% level. Overall, error rates did not vary appreciably as a function of condition or case. For same-case displays, mean error rates for CAWR, COR, CONT, and CMPW trials were $1.4 \%$, $1.9 \%, 2.4 \%$, and $2.0 \%$ of all trials, respectively, whereas the respective values for different-case displays were $1.6 \%, 1.7 \%, 2.8 \%$, and $2.4 \%$.

The relative facilitation for CAWR and COR trials suggests that correct color names for S1 were highly available and facilitated responding for S2. The relative impairment for CMPW sequences indicates that the incorrect, distractor-activated responses for $\mathrm{S} 1$ were especially difficult to report. The pattern of results closely parallels patterns reported previously (Lowe, 1979; Neill, 1977) in demonstrating both repetition and suppression effects. A major difference between the current results and those earlier findings, however, concerns the failure to obtain faster RTs for CAWR trials than for COR trials. The absence of identity effects indicates that the use of mixed lists successfully discouraged the continued maintenance of S1 word-forms for comparison with S2 (Posner et al., 1969). More important, the absence of identity effects, in the context of supression effects, contradicts the notion that physical matching is the common source of identity and suppression effects (Lowe, 1979). Accord- ing to that idea, suppression effects should not have occurred unless accompanied by identity effects. These findings make it clear that, although the identity effect is tied closely to a strategy of maintaining the physical code of $\mathrm{S} 1$, physical matching cannot adequately explain the suppression effect.

\section{EXPERIMENTS 2 AND 3}

The results of Experiment 1 documented the independence of identity and suppression effects. However, it is puzzling that identity effects were not obtained for samecase displays, since the results of Posner et al. (1969) had illustrated that, even for mixed lists, RTs for physically similar inputs were relatively facilitated. Possibly, the use of mixed lists in the current paradigm especially impaired subjects' attempts to compare successive word-forms.

In order to confirm the results of Experiment 1, identity and suppression effects were examined in both pure (Experiment 2) and mixed (Experiment 3) lists. In these experiments, S1 was presented briefly and was followed by variable intervals before $\mathbf{S} 2$ onset. By manipulating stimulus onset asynchrony (SOA), it is possible to vary the time available for selectively processing $S 1$ before the necessity of attending S2. As noted, Keele (1973) suggested that, in order to determine which of the competing color names should be reported, subjects must determine from visual information which matches the color and which corresponds to the word. In the present paradigm, subjects may first attend the available ink-color and wordform information, and therefore identity effects may be observed at briefer SOAs when subjects are engaged with the physical properties of S1. Thus, for mixed lists, identity effects may be obtained for same-case displays but not for different-case displays. However, if at longer SOAs, having successfully sorted out the competing responses for S1, subjects actively maintain only correct color-activated responses for comparison with $\mathbf{S} 2$, then identity effects may be eliminated for both similar- and dissimilar-case displays. Lastly, if pure lists provide the incentive for subjects to continuously maintain the physical code of S1, identity effects may occur regardless of changes in SOA. Such a pattern of results would confirm the suggestion that the use of mixed lists especially discouraged the continued maintenance of $\mathrm{S} 1$ word-form information.

A second purpose of Experiments 2 and 3 was to chart the time course of identity and suppression effects. According to the premise that subjects might first attend the available physical codes, suppression effects may be obtained only after that process is complete. Thus, whereas identity effects may occur primarily at earlier SOAs, suppression effects may be restricted to later intervals, when the results of the analysis of the visual information are available. Such a pattern of results, revealing asymmetric time courses in the onsets of identity and suppression effects, would provide additional evidence regarding their independence. 


\section{Method}

General. The methods and procedures were basically the same as those of Experiment 1. In Experiment 2, pure lists of uppercase stimuli were employed; in Experiment 3, mixed lists were used. In Experiment 2, for each SOA, $75 \%$ of all trials consisted of CAWR and COR $(37.5 \%$ each) trials; the remaining trials were composed of CONT and CMPW sequences ( $12.5 \%$ each). In Experiment 3, for each SOA. for both similar- and dissimilar-case displays, CAWR and COR sequences constituted $18.75 \%$ of all trials: the balance consisted of CONT and CMPW trials ( $6.25 \%$ each). For both experimetns, SOAs of $50,100,200$, or $400 \mathrm{msec}$ were composed of 50 -msec $S 1$ presentations followed by variable intervals, during which the preexposure field was presented for 0.50 , 150 , or $350 \mathrm{msec}$, respectively, before $\mathrm{S} 2$ onset. In both experiments, all subjects served in all conditions for four consecutive daily sessions. Data from the first session were discarded as practice. Within each session, the different SOAs were presented in blocks of 48 trials. The order in which SOAs were administered to individual subjects was determined randomly and presented in a counterbalanced fashion. The subjects were instructed not to respond to $\mathrm{S} 1$, but to focus on its color and to respond to $\mathrm{S} 2$ after its onset. All other aspects were identical to Experiment 1.

Subjects. The six subjects in each experiment were selected from the same population as those subjects in Experiment 1 and were paid $\$ 12.00$ each for their participation.

\section{Results and Discussion}

Performance scores were calculated as in Experiment 1, except that, for each SOA, median RTs for those conditions constituting $6.25 \%, 12.5 \%, 18.75 \%$, and $37.5 \%$ of all trials were based on $18,36.54$, and 108 , or fewer, correct trials, respectively. Error rates were relatively low (1.9\% and $2.1 \%$ of all trials for Experiments 2 and 3. respectively) and did not vary appreciably as a function of condition, case, or SOA. The RT scores for Experiment 2 were submitted to a $4 \times 4$ repeated-measures analysis of variance to evaluate conditions and SOAs. For Experiment $3,4 \times 2 \times 4$ repeated-measures analysis of variance evaluated conditions. case. and SOAs.

Analysis of the results of Experiment 2. displayed in the leftmost panel of Figure 1, revealed that there were large effects upon RTs as a function of condition $\mid \mathrm{F}(3,15)$

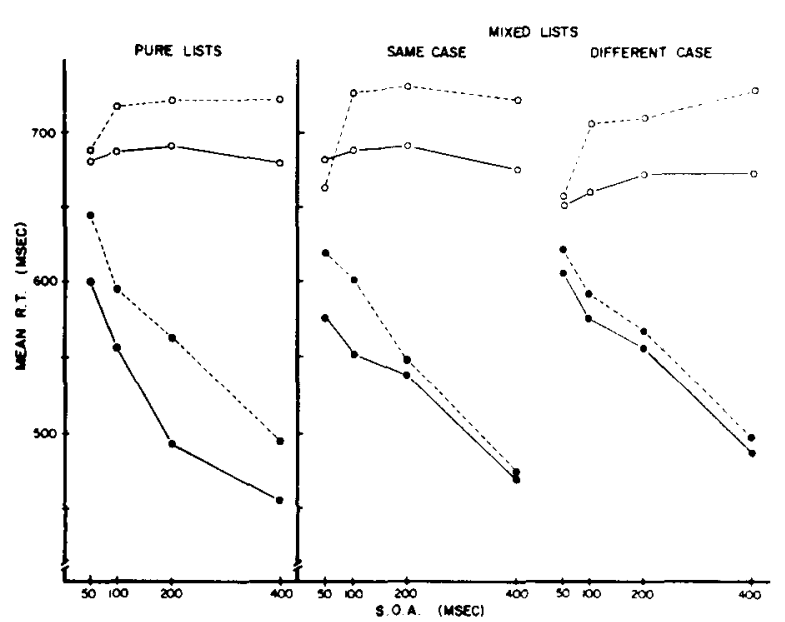

Figure 1. Mean RTs for Experiments 2 (pure lists) and 3 (mixed lists). Color and word repeated $=\bullet-\bullet$; color only repeated $=$ $\bullet-\cdots$; control (unrelated) $=0 \_$; color matches preceding word $=0--0$.
$=219.76, \mathrm{MSe}=700]$. Overall RTs for CAWR, COR, CONT, and CMPW sequences were 526, 569, 668, and $695 \mathrm{msec}$, respectively. Newman-Keuls comparisons of the four means revealed that repetition, identity, and suppression effects were obtained; all conditions differed from each other at the $1 \%$ level. Increases in SOA produced faster overall $\mathrm{RTs}[\mathrm{F}(3,15)=31.75$, MSe $=$ 657], in that average latencies ranged from a high of $644 \mathrm{msec}$ at the 50 -msec SOA to a low of $576 \mathrm{msec}$ at the $400-\mathrm{msec}$ SOA. The analysis also revealed a reliable condition $\times$ SOA interaction $[\mathrm{F}(9,45)=20.74, \mathrm{MSe}=$ 450]. It is evident from Figure 1 that performance on CONT trials was virtually unaffected by changes in SOA. However, as SOA increased, RTs for both CAWR and COR sequences progressively improved, but, by the 100msec SOA and beyond, latencies for CMPW trials were relatively impaired. Separate Newman-Keuls tests on the means of the four conditions at each SOA revealed the following patterns: (1) at the 50-msec SOA, (CAWR), (COR), (CONT, CMPW), and (2) at the 100-msec SOA and beyond, (CAWR), (COR), (CONT), (CMPW), where only conditions within the same parentheses did not differ at the $5 \%$ level. Thus, repetition and identity effects were obtained at all SOAs employed. In contrast. suppression effects were not evident until the $100-\mathrm{msec}$ SOA.

An inspection of the rightmost panels of Figure 1 reveals that the results of Experiment 3 were basically similar. The effects of condition $[\mathrm{F}(3,15)=36.31, \mathrm{MSe}=$ $7,124]$ and $\operatorname{SOA}[\mathrm{F}(3,15)=14.83$, MSe $=1,624]$, and their interaction $[\mathrm{F}(9,45)=31.02, \mathrm{MSe}=508]$, were all statistically reliable. As SOA increased, performance for both CAWR and COR sequences progressively improved, and, by the 100-msec SOA, latencies for CMPW trials were impaired. The analysis also revealed that the effects of case were not significant $[\mathrm{F}(1,5)<1$, MSe $=$ 1,837 ] and that case did not interact reliably with either condition $[\mathrm{F}(3,15)=1.84, \mathrm{MSe}=903]$ or $\mathrm{SOA}[\mathrm{F}(3,15)$ $=2.15, \mathrm{MSe}=320 \mathrm{j}$. There was, however, a reliable case $\times$ conditions $\times$ SOA interaction $[F(9.45)=3.7$. $\mathrm{MSe}=383 \mathrm{~J}$. As in Experiment 2. RTs for CAWR trials were faster than those for COR sequences, but only for same-case displays. In contrast to the results of Experiment 2, however, the advantage for identical inputs was restricted to SOAs of 50 and $100 \mathrm{msec}$. For different-case displays, RTs for CAWR trials were not substantially faster than those for COR trials at all SOAs employed. For same-case displays, separate Newman-Keuls comparisons of the means of the conditions at each SOA revealed the following patterns: (1) at the 50-msec SOA, (CAWR), (COR), (CONT, CMPW); (2) at the 100-msec SOA, (CAWR), (COR), (CONT), (CMPW); and (3) at the 200- and 400-msec SOAs. (CAWR. COPR), (CONT), (CMPW). Similar tests performed for different-case displays indicated: (1) at the 50-msec SOA. (CAWR.COR). (CONT. CMPW): and (2) at the 100-msec SOA and beyond. (CAWR. COR). (CONT), (CMPW).

The pattern of results illustrates that. in mixed lists, identity effects were obtained for same-case displays, but 
only at brief processing intervals. In contrast, for pure lists, identity effects were obtained at all SOAs employed. The results of Experiment 3 confirm the findings of Experiment 1 and support the suggestion that the use of mixed lists discouraged the continued maintenance of $\mathrm{S} 1$ distractor-activated information, especially as SOA was increased. It thus appears that, in Experiment 1, the S1S2 SOA (i.e., the time required for subjects to respond to $S 1$, thereby initiating $S 2$ onset) was sufficient to impair the comparison of successive word-forms. Thus, for mixed lists, the subjects apparently preferred to maintain only correct color-activated responses for $\mathrm{S} 1$ as processing time was extended. However, for pure lists, it seems that both correct color responses and incorrect distractor word-forms for $\mathrm{S} 1$ were continuously maintained for comparison with S2.

These findings provide additional evidence regarding the dissociation of identity and suppression effects. In both experiments, identity effects were obtained as early as the 50-msec SOA, whereas suppression effects were not manifested until the 100-msec SOA. Since the onsets of these phenomena display asymmetric time courses, it seems safe to conclude that physical matching of distractor-activated information, which is the likely source of identity effects, cannot be responsible for suppression effects. If physical matching were the basis of both events (Lowe, 1979) it might be expected that the time of their onsets would be similar.

The current results parallel the findings of recent experiments employing the priming paradigm. In the priming paradigm, subjects are required to make a judgment about a target stimulus (S2), which is preceded by a priming stimulus (S1). The effects of varying the validity of $\mathrm{S} 1$ in predicting some aspect of $\mathrm{S} 2$ have been investigated by Neely $(1976,1977)$ and Posner and Snyder (1975). The results indicated that RTs are facilitated when S1-S2 relations are consistent with subjects' predictions and are inhibited when they are not. In those experiments, the S1S2 SOA was also varied, and the results revealed that the delay must be greater than $200-500 \mathrm{msec}$ for facilitation and inhibition to have their strongest effects.

These findings from the priming paradigm are generally consistent with the facilitatory effects obtained for expected CAWR and COR trials, but the onset of the inhibitory effects for unexpected CMPW trials occurred by $100 \mathrm{msec}$ of delay. However, it is also known that inhibition can occur very early and is relatively constant across longer SOAs whenever stimulus priming and response repetition are possible (Posner, 1978). Since, in the current paradigm, it is both the ink color and the response that were likely to be repeated from $S 1$ to $S 2$, the time courses of both facilitation and inhibition as obtained here seem to correspond to those from the priming paradigm.

\section{GENERAL DISCUSSION}

The results of these experiments demonstrate a suppression effect. When the color of a conflicting color word is named, RT is slowed if the color has the same name as the color word of the preceding stimulus. Earlier, Lowe (1979) explained this effect by a physical matching strategy in which, under certain circumstances, subjects compared the physical attributes of successive stimuli. However, the present results rule out that strict interpretation, since the suppression effect occurs independently of physical identity effects. Nonetheless, the findings reveal that, in the current paradigm, matching strategies can play a role, since subjects will compare various aspects of the sequential displays when they are encouraged to do so. As such, physical matching was a plausible account that needed to be assessed.

How may the suppression effect be explained, and why is it so sensitive to contextual variations? Two hypotheses may be entertained-an inhibitory hypothesis (Neill, 1977) and a code-coordination hypothesis (Keele \& Neill, 1978; Treisman \& Gelade, 1980). On one hand, the suppression effect may truly index attentional inhibition. There is abundant evidence that upon appearance of a stimulus, multiple information codes stored in memory-physical (Keele \& Neill, 1978), orthographical (Tanenhaus, Flanigan, \& Seidenberg, 1980), phonemic (Meyer, Schvaneveldt, \& Ruddy, 1975), semantic (Conrad, 1974)-are automatically accessed. This relatively passive and broad activation requires subsequent attentional selection and coordination of information (Keele \& Neill, 1978; Treisman \& Gelade, 1980). Thus, as some codes are selected and passed along for further processing, perhaps other messages are inhibited and ultimately are not retained. Therefore, in the Stroop task, perhaps both colors and words activate, among other things, their visual and phonemic representations. According to the inhibitory theory (Neill, 1977), an attentional process then selects and combines the relevant, coloractivated codes, while suppressing the conflicting wordactivated memories. Then, if that inhibited information is the correct response for $\mathbf{S 2}$, the inhibition slows responding, producing the suppression effect.

However, it is also possible that the occurrence of the suppression effect may be governed by particular experimental details. For example, Neill $(1977,1979)$ suggested that inhibition may be controlled by the degree of discriminability required between relevant and irrelevant messages. Thus, suppression may be governed by the amount of interference that the irrelevant information produces. Therefore, in the Stroop task, when vocal responding is demanded, accuracy of report is required, and difficult color-word stimuli are encountered trial after trial, the interference may be so extreme that inhibition of distractor-activated information is the best method to cope with the prevailing conditions. In these exceptional circumstances, selection of color-activated memories, although leaving the competing word-activated codes highly available could permit too many conflicting color names to persist during subsequent trials. Under such conditions, the task may become unmanageable. However, in less taxing environments, such as those in which (1) simpler, easy-to-name, color patches are sometimes 
encountered, (Lowe, 1979, Experiments 3 and 4), (2) less Stroop interference is experienced during manual responding (Keele, 1972; Neill, 1977, Experiment 2) or (3) accuracy of report is not important (Neill, 1977, 1979), relevant color-activated information may be selected, while competing messages are left unhampered.

The existence of inhibitory mechanisms in the context of selective attention may be useful in understanding the findings of recent experiments that used the priming paradigm to study recognition of polysemous words. There is consistent evidence that, immediately on occurrence of a lexically ambiguous word, multiple meanings of that word may be activated briefly. Supporting evidence comes from experiments using three different response measures: (1) pronunciation (Seidenberg, Tanenhaus, Leiman, \& Bienkowski, 1982; Tanenhaus, Leiman, \& Seidenberg, 1979), (2) lexical decision (Holley-Wilcox \& Blank, 1980; Swinney, 1979), and (3) color naming (Conrad, 1974; Oden \& Spira, 1983). Pronunciation latencies and lexical decisions to target words related to only one of the meanings of a preceding ambiguous word are facilitated, and interference in color naming occurs. In addition, the results of experiments that have varied the prime-target SOA have revealed that the availability of the alternate meanings of an ambiguous word changes over time. For example, the results of studies by Seidenberg et al. (1982), Swinney (1979), and Tanenhaus et al. (1979), showed that, at brief intervals following prime onset, subjects accessed multiple readings of an ambiguous word, since both meanings of that word were primed. However, at longer delays (i.e., within a few hundred milliseconds), only one meaning of an ambiguous word was primed, whereas alternate readings were not facilitated. Because multiple meanings were available at the earlier SOA, it was argued that a single meaning of the word was rapidly selected and that inhibition of all but that single reading must have occurred (Seidenberg et al., 1982).

However, this inhibition hypothesis seems inadequate for the present Stroop test results: Lowe (1979, Experiment 4) studied a case in which S1 consisted of a colorword stimulus that was followed by unpredictable presentations of another color-word stimulus or by just a color patch (i.e., no conflicting color word). Lowe still obtained the suppression effect when color-word items served as S2. If this result means that the name of the competing response for S1 had been inhibited, then it would be expected that RTs would also be impaired when S2 was just a color patch. However, RTs were actually facilitated when $\mathrm{S} 2$ was a color patch, indicating that $\mathrm{S} 1$ incorrect responses remained available and primed correct color responses for S2. Thus, activation or inhibition of S1 conflicting information can occur within the same observer when the nature of $\mathrm{S} 2$ is unpredictable. Therefore, an inhibition explanation of the suppression effect seems untenable.

Lowe (1979) argued that the supression effect can be attributed to the ambiguity of correct color responses for
S2. That is, on presentation of $\mathbf{S} 2$, two potential responses are highly activated and subjects must decide which corresponds to the color. On those occasions when the correct color response for $\mathrm{S} 2$ matches both the current color and the preceding word, subjects are slower to respond, not because the inappropriate response for $\mathrm{S} 1$ has been inhibited, but, rather, because the S1 word persists and the subjects exhibit some confusion over whether the correct color name is from the current color rather than from the preceding word.

There are several ways to characterize the nature of that confusion. First, the suppression effect may arise because of the implicit comparison and the resulting conflict between successive incorrect and correct color-name responses. However, an explanation in terms only of conflict arising from successive withholding and emission of the same response seems to be inadequate. For example, such an account cannot explain why conflict does not also arise, thus yielding a suppression effect, when color patches are substituted for color-word stimuli as S2 (Lowe, 1979, Experiments 3 and 4). In addition, it might also be expected that a condition that requires successive emission and withholding of the same response, as when S2's word matches S1's color, should also give rise to confusion, thus yielding impaired performance. However, results obtained by Dalrymple-Alford and Budayr (1966, Experiment 2), Lowe (1979, Experiment 1), and Neill (1978. Experiment 1) have demonstrated that RTs for such word-matches-preceding-color trials are actually faster than latencies for both CONT and CMPW sequences.

Thus, as noted earlier, an alternative hypothesis to explain the suppression effect is that of code coordination. According to the idea that relevant information must be selected and integrated into a final response (Keele \& Neill, 1978), perhaps both the color- and word-activated memories must be properly combined before color naming is possible in the Stroop task. Therefore, having successfully aligned the correct and incorrect color names for SI with their visual codes, it is possible that, during CMPW trials, subjects experience problems in properly coordinating the relevant color-activated codes for $\mathbf{S} 2$. As suggested, correct color responses for $\mathrm{S} 2$ would be simultaneously activated by both the current correct color and the persisting incorrect word.

If this notion is accepted, it follows that suppression effects might not be expected to occur whenever experimental context makes integration of the available memory codes for $\mathrm{S} 2$ unnecessary. These circumstances would be fulfilled when irrelevant color names are not activated by $\mathrm{S} 2$, as when color patches are substituted for color-word stimuli (Lowe, 1979, Experiments 3 and 4). Code integration would also be unnecessary, thus eliminating suppression effects, when manual responding is permitted (Neill, 1977, Experiment 2). During manual responding. correct responses may be based directly on the colors of the displays, while naming mechanisms are bypassed altogether (Azkoul, as cited in 
Dalrymple-Alford \& Azkoul, 1972). Finally, it would also be expected that, in the present experiments, suppression effects would be obtained regardless of variations in the fonts of the stimuli. For both same- and different-case displays, code integration would be necessary for S2 before color naming would be possible. In this situation, since the $\mathbf{S} 1$ word persists, correct color names for S2 during CMPW trials would be ambiguous whether the sequential distractors were physically similar or dissimilar.

\section{REFERENCES}

Bertelson, P. (1963). S-R relationships and reaction times to new versus repeated signals in a serial task. Journal of Experimental Psychology, 65, 478-484.

ConRad, C. (1974). Context effects in sentence comprehension: A study of the subjective lexicon. Memory \& Cognition, 2, 130-138.

Dalrymple-Alford, E. C. , \& AzKoul, J. (1972). The locus of interference in the Stroop and related tasks. Perception \& Psychophysics, $11,385-388$.

DALRYMPLE-AlFord, E. C., \& BUDAYr, B. (1966). Examination of some aspects of the Stroop color-word test. Perceptual and Motor Skills, 23, $1211-1214$.

Holley-Wilcox, P., \& Blank, M. (1980). Processing ambiguous words: Evidence for multiple access. Journal of Experimental Psychology: Human Perception and Performance, 8, 416-432.

KEELE, S. W. (1972) Attention demands of memory retrieval. Journal of Experimental Psychology, 93, 245-248.

KeELE, S. W. (1973). Attention and human performance. Pacific Palisades, CA: Goodyear.

KeEle, S. W., \& NeILl, W. T. (1978). Mechanisms of attention. In E. C. Carterette \& P. Friedman (Eds.), Handbook of perception (Vol. 9). New York: Academic Press.

LowE, D. G. (1979). Strategies, context, and the mechanism of response inhibition. Memory \& Cognition, 7, 382-389.

Meyer, D. E., Schvaneveldt, R. W., \& Ruddy, M. G. (1975). Loci of contextual effects on visual word recognition. In P. M. A. Rabbitt \& S. Dornic (Eds.), Attention and performance V. New York: Academic Press.

NeELy, J. H. (1976). Semantic priming and retrieval from lexical memory: Evidence for facilitatory and inhibitory processes. Memory \& Cognition, 4, 648-654.

NeELy, J. H. (1977). Semantic priming and retrieval from lexical memory: Roles of inhibitionless spreading activation and limited capacity attention. Journal of Experimental Psychology: General, 106, 226-254.

NeILL, W. T. (1977). Inhibitory and facilitatory processes in selective attention. Journal of Experimental Psychology: Human Perception and Performance, 3, 444-450.
NeILL, W. T. (1978). Decision processes in selective attention: Response priming in the Stroop color-word task. Perception \& Psychophysics, 23, 80-84.

NeILL, W. T. (1979). Switching attention within and between categories: Evidence for intracategory inhibition. Memory \& Cognition, 7, 283-290.

ODEN, G. D., \& SPIRA, J. L. (1983). Influence of context on the activation of ambiguous word senses. Quarterly Journal of Experimental Psychology, 35, 51-64.

POSNER, M. I. (1978). Chronometric explorations of mind. Hillsdale, $\mathrm{NJ}$ : Erlbaum.

Posner, M. I., Boies, S. J., Eichelman, W. H., \& Taylor, R. H. (1969). Retention of visual and name codes of single letters. Journal of Experimental Psychology Monographs, 79(1, Pt. 2).

Posner, M. I., \& Mitchell, R. F. (1967). Chronometric analysis of classificaiton. Psychological Review, 74, 392-409.

POSNER, M. I., \& SNYDER, C. R. R. (1975). Attention and cognitive control. In R. L. Solso (Ed.), Information Processing and Cognition: The Loyola Symposium. Hillsdale, NJ: Erlbaum.

Seidenberg, M. S., Tanenhaus, M. K., Leiman, J. M., \& BIENKowsKI, M. (1982). Automatic access of the meanings of some ambiguous words: Some limitations of knowledge-based processing. Cognitive Psychology, 14, 489-537.

STROOP, J. R. (1935). Studies of interference in serial verbal reactions. Journal of Experimental Psychology, 18, 643-661.

SwinNey, D. A. (1979). Lexical access during sentence comprehension: $(\mathrm{Re})$ consideraton of context effects. Journal of Verbal Learning and Verbal Behavior, 18, 523-534.

Tanenhaus, M. K., Flanigan, H., Seidenberg, M. S. (1980). Orthographic and phonological code activation in auditory and visual word recognition. Memory \& Cognition, 8, 513-520.

Tanenhaus, M. K., Leiman, J. M., \& Seidenberg, M. S. (1979). Evidence for multiple stages in the processing of ambiguous words in syntactic contexts. Joumal of Verbal Leaming and Verbal Behavior, $18,427-440$.

Treisman, A. M. (1964). Verbal cues, language, and meaning in selective attention: American Joumal of Psychology, 77, 206-219.

Treisman, A. M., \& Gelade, G. (1980). A feature-integration theory of attention. Cognitive Psychology, 12, 97-136.

\section{NOTES}

1. Strictly speaking, of course, different-case displays cannot be identical. The terminology is employed only for continuity and ease of exposition.

2. The rejection region for all statistical tests reported in this paper is $\mathrm{p}<.05$ or better.

(Manuscript received July 24, 1984; revision accepted for publication November 2, 1984.) 